\title{
Mixed Topology of DF Relayed Terrestrial Optical Wireless Links with Generalized Pointing Errors over Turbulence Channels
}

\author{
George K. Varotsos ${ }^{1}{ }^{1}$, Hector E. Nistazakis ${ }^{1, *}$, Argyris N. Stassinakis ${ }^{1}$, Christos K. $\operatorname{Volos}^{2}{ }^{\mathbb{D}}$, \\ Vasileios Christofilakis ${ }^{3}$ and George $S$. Tombras ${ }^{1}$ \\ 1 Department of Electronics, Computers, Telecommunications and Control, Faculty of Physics, National and \\ Kapodistrian University of Athens, 15784 Athens, Greece; georgevar@phys.uoa.gr (G.K.V.); \\ a-stasinakis@phys.uoa.gr (A.N.S.); gtombras@phys.uoa.gr (G.S.T.) \\ 2 Laboratory of Nonlinear Systems, Circuits \& Complexity, Physics Department, Aristotle University of \\ Thessaloniki, 54124 Thessaloniki, Greece; volos@physics.auth.gr \\ 3 Physics Department, Electronics-Telecommunications and Applications Laboratory, University of Ioannina, \\ 45110 Ioannina, Greece; vachrist@uoi.gr \\ * Correspondence: enistaz@phys.uoa.gr; Tel.: +30-210-727-6710
}

Received: 1 November 2018; Accepted: 12 December 2018; Published: 17 December 2018

\begin{abstract}
This study investigated the outage performance of a terrestrial FSO communication system that uses mixed series and parallel decode-and-forward (DF) relay-assisted (i.e., cooperative diversity) configurations, taking into account the influence of both atmospheric turbulence and pointing error effects. Turbulence-induced optical signal fading is modeled by gamma-gamma or the negative exponential distribution for weak to strong and saturated turbulence conditions, respectively. Additionally, weak to strong non-zero boresight misalignment-induced optical signal fading is modeled by the generalized Beckmann distribution. Under these conditions, an outage analysis of the examined FSO system is performed, in terms of both outage probability and mean outage duration metrics. Thus, fairly accurate closed-form mathematical expressions for both performance metrics are derived, while their corresponding analytical results demonstrate concrete performance and availability improvements for the total FSO system, especially when the number of the connected in parallel DF relays increases. Moreover, the obtained results are verified through the corresponding simulation results.
\end{abstract}

Keywords: free space optical communication systems; mixed series and parallel DF relay-assisted configurations; nonzero boresight misalignment-induced fading; turbulence-induced fading; gamma-gamma distribution; negative exponential distribution; Beckmann distribution; outage probability; mean outage duration

\section{Introduction}

Terrestrial FSO communication systems that establish line-of-sight optical wireless links by using lasers and photo-detectors as transmitter and receiver terminals, respectively, have had growing research and commercial attention in the last few years. This is mainly due to the very high capacity, along with a very high security level, they can achieve, their operation with low power consumption in the unlicensed optical spectrum, their relatively low operational and installation costs, their flexibility for deployment and redeployment, their immunity to electromagnetic interference and multi-path dispersion, and their compatibility with the very performance-effective optical fiber communication systems [1-4]. 
Nevertheless, even in clear weather conditions and particularly over propagation distanceranges of the order of $1 \mathrm{~km}$ or longer, atmospheric turbulence attenuates the propagating signal and causes the so-called scintillation effect, which results in random variations of the received signal irradiance that significantly degrades the FSO performance and availability [4,5]. Additionally, wind loads, earthquakes and thermal expansion of building frame parts bring about the sway of high-rise buildings, where the FSO terminals are usually installed. Therefore, the line of sight alignment between transmitter and receiveris diminished by building sway, which results in misalignment-induced fading of the received signal irradiance, commonly known as the pointing errors effect [6,7]. Here, the pointing errors effect is investigated by taking into account both the fixed displacement between the beam center and center of the detector, which is called the boresight, and the random spatial jitter as well $[8,9]$.

All the above-mentioned effects significantly degrade the system's performance. Thus, in order to counterbalance their negative side-effects, many techniques have been proposed. One such effective technique is the use of relays, which broadens the useful coverage area of the optical wireless communication system and upgrades the link's performance characteristics [10-17]. The relays can be used either in serial or parallel configuration, according the needs of the specific FSO system. More specifically, the serially relayed optical links can be used for the cases where the extension of the link length is the main requirement, while parallel configuration is more useful when the reliability and the availability of the link need to be improved. Consequently, a mixed architecture with parallel and serial relayed FSO links can significantly improve the performance of the system [17]. In this context, a recently published work [17] estimated the outage probability (OP) of a mixed decode-and-forward (DF) FSO system over weak turbulent channels, modeled through the compact gamma distribution [18], along with non-zero boresight pointing errors modeled, in turn, with the accurate approximation of Beckmann's distribution [19].According to the mixed DF FSO topology proposed in [17], the source node initiallytransmitsthe same signal to a specific number of DF relay nodes in a parallel configuration. Next, each one of them decodes the signal after direct detection, modulates it withon-off keying (OOK), and retransmits it to a specific number of DF relay nodes in a serial configuration. Thus, at the destination node's side, as many copies of the same information signal as the number of the parallel multi-hop branches of thespecific mixed FSO systemare eventually arriving. In this way, it enhancesboth the reliability and the useful coverage area of the total FSO system.

Motivated from the above, in this work we estimate, under weak to saturated turbulence channels, the outage performance of the link by means of both OP and mean outage duration time (MOD) metrics for various mixed DF relay-assisted FSO system configurations, along with the presence of non-zero boresight pointing errors. In this respect, weak to strong turbulence is modeled through the well-known gamma-gamma(G-G) distribution $[3,18,20]$, while saturated turbulence is described through the proper negative exponential (NE) distribution [21,22], respectively. Moreover, non-zero boresight pointing errors are emulated through the accurate approximation of the Beckmann distribution, proposed in [19].

\section{System and Channel Model}

\subsection{Signal and System Assumptions}

The proposed mixed DF relay-assisted FSO system transmits the same information-bearing optical signal through $N$ different parallel paths. Additionally, each path employs $H-1$ serially connected DF relays, with $H \geq 1$, and thus each of the $N$ paths consists of $H$ intermediate links, commonly known as hops, as can be seen in Figure 1 . Thus, the specific case of $N=1$ describes the single input single output (SISO) link configuration, which is either a single-hop or a multi-hop one, for $H=1$ and $H>1$, respectively. Similarly, for the generalized case of $N>1$ along with $H>1$, the system is considered as a mixed single input-multiple output (SIMO) and multiple input single output (MISO) one, respectively. Indeed, from the transmitter to each first DF relay node of each parallel path, the system consists of one source (i.e., the transmitter) and $N$ different destinations (i.e., the $N$ parallel first relay nodes), 
and thus, it can be considered as a SIMO one. Then, each DF relay decodes its received signal's copy and retransmits it to the next, serially connected DF relay of the same path. This procedure continues until the final retransmissions, i.e., from the final DF relay nodes to the final receiver node of the total system. Thus, from the last DF node of each parallel path to the final receiver node, the system consists of multiple transmitter DF nodes and one receiver node, i.e., this part of the system is a multiple input single output (MISO) one. It is notable that we consider OOK signaling with an intensity modulation and direct detection (IM/DD) format in order to overcome any interference of the arriving information signal copies, from different paths, at the receiver node of the total system. Under these assumptions, the received signal, $y_{\{i, j\}}$, at the $j$ th hop in the $i$ th path is expressed as [10,15]:

$$
y_{\{i, j\}}=\eta I_{\{i, j\}} x_{\{i, j\}}+n_{\{i, j\}}
$$

where $i$ indicates a specific parallel path, i.e., $i=\{1,2 \ldots N\}, j$ represents a specific hop in the parallel path, i.e., $j=\{1,2 \ldots H\}, \eta$ stands for the effective photo-current conversion ratio, $I_{\{i, j\}}$, is the normalized irradiance in the $j$ th hop in the $i$ th path, while $x_{\{i, j\}}$ and $n_{\{i, j\}}$ denote the corresponding modulated signal and the additive white Gaussian noise (AWGN) with zero mean and variance $N_{0} / 2$, respectively $[3,4]$.

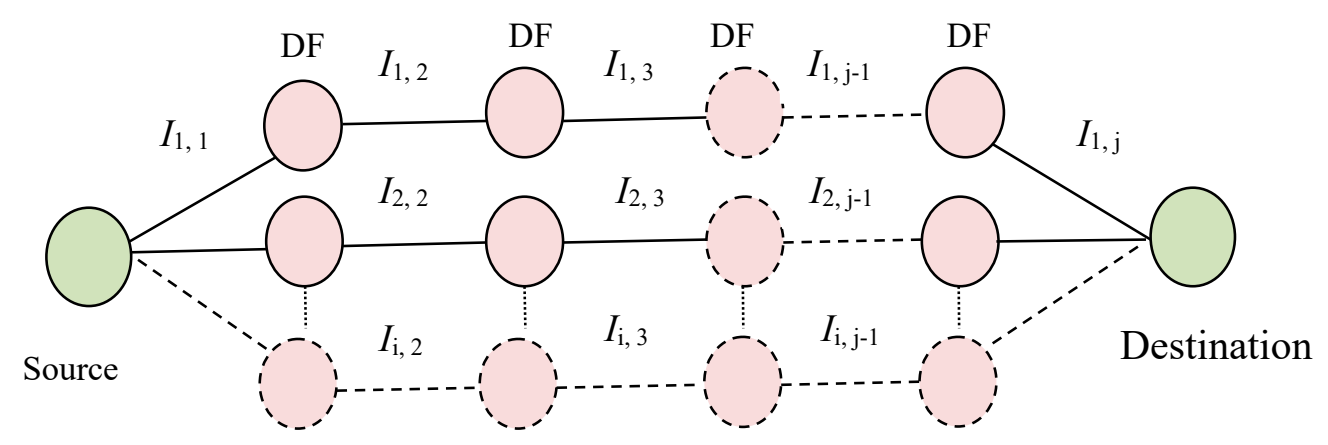

Figure 1. Mixed decode-and-forward (DF) relayed FSO communication system.

Owing to atmospheric turbulence, pointing errors and attenuation effects, the normalized irradiance, $I_{\{i, j\}}$, is written as $[3,4,10]$ :

$$
I_{\{i, j\}}=I_{a,\{i, j\}} I_{p,\{i, j\}} I_{l,\{i, j\}}
$$

where $I_{a,\{i, j\}}$ and $I_{p,\{i, j\}}$ represent the normalized received irradiance in the $j$ th hop in the $i$ th path due to turbulence-induced and misalignment-induced fading, respectively, while $I_{l,\{i, j\}}$ stands for the corresponding deterministic weather-dependantpath loss parameter, [23]. However, the investigation of the path losses is not the scope of this work and thus, without loss of generality, it is assumed that is normalized to unity $\left(I_{l, m}=1\right)[4,24]$.

\subsection{The Atmospheric Turbulence Effect}

By assuming weak to strong turbulence conditions, the G-G distribution is used. Thus, the corresponding probability density function (PDF), as a function of the irradiance, $I_{a,\{i, j\}}$, is given as $[3,20]$ :

$$
\begin{aligned}
f_{G G, I_{a,\{i, j\}}}\left(I_{a,\{i, j\}}\right) & =\frac{2(\alpha \beta)^{\frac{\alpha+\beta}{2}}}{\Gamma(\alpha) \Gamma(\beta)} I_{a,\{i, j\}}^{\frac{\alpha+\beta}{2}-1} \\
& \times K_{\alpha-\beta}\left(2 \sqrt{\alpha \beta I_{a,\{i, j\}}}\right), \quad I_{a,\{i, j\}}>0
\end{aligned}
$$


where $K_{v}($.$) denotes the modified Bessel function of the second kind of order v$ (Equation (8.432.2) in [25]), $\Gamma$ (.) represents the gamma function (Equation (8.310.1) in [25]), and the parameters $\alpha, \beta$ are estimated from each link's parameters through the following expressions [22,26]:

$$
\begin{aligned}
& \alpha=\left[\exp \left(\frac{0.49 \delta^{2}}{\left(1+0.18 d^{2}+0.56 \delta^{12 / 5}\right)^{7 / 6}}\right)-1\right]^{-1} \\
& \beta=\left[\exp \left(\frac{0.51 \delta^{2}\left(1+0.69 \delta^{12 / 5}\right)^{-5 / 6}}{\left(1+0.9 d^{2}+0.62 d^{2} \delta^{12 / 5}\right)^{5 / 6}}\right)-1\right]^{-1}
\end{aligned}
$$

where $d=0.5 D \sqrt{2 \pi \lambda^{-1} L^{-1}}, \delta^{2}=0.5 C_{n}^{2} \kappa^{7 / 6} L^{11 / 6}, \kappa=2 \pi / \lambda$, with $D$ representing the receiver's aperture diameter, $\lambda$ the operational wavelength, $L$ the link's length and $C_{n}{ }^{2}$ the refractive index structure parameter which is proportional to the atmospheric turbulence strength, and varies between $10^{-17} \mathrm{~m}^{-2 / 3}$ and $10^{-13} \mathrm{~m}^{-2 / 3}$ for weak to strong turbulence, respectively [27].

Additionally, by assuming saturated turbulence conditions, the negative exponential is the most suitable model and its PDF, as a function of $I_{a,\{i, j\}}$, is given as $[21,22,28]$ :

$$
f_{N E, I_{a,\{i, j\}}}\left(I_{a,\{i, j\}}\right)=1-\exp \left(-I_{a,\{i, j\}}\right), I_{a,\{i, j\}}>0
$$

\subsection{Generalized Pointing Errors}

A generalized and realistic statistical model, which accurately describes the pointing errors effect, considering the influence of beam width, the detector's size, the different jitters for the elevation and the horizontal displacement, as well as the effect of non-zero boresight errors, is the Beckmann's distribution [9], which is approximated as [19]:

$$
f_{R_{\{i, j\}}}\left(R_{\{i, j\}}\right)=\frac{R_{\{i, j\}}}{\sigma_{\bmod ,\{i, j\}}^{2}} \exp \left(-\frac{R_{\{i, j\}}^{2}}{2 \sigma_{\bmod ,\{i, j\}}^{2}}\right), \quad R_{\{i, j\}} \geq 0
$$

With $R_{\{i, j\}}$ being the radial displacement in the $j$ th hop in the $i$ th path that is obtained as $R_{\{i, j\}}=\left|\vec{R}_{\{i, j\}}\right|=\sqrt{R_{x,\{i, j\}}^{2}+R_{y,\{i, j\}}^{2}}$, while $\vec{R}_{\{i, j\}}=\left[R_{x,\{i, j\}}, R_{y,\{i, j\}}\right]^{T}$ stands for the radial displacement vector, with $R_{x,\{i, j\}}$ and $R_{y,\{i, j\}}$ representing the corresponding offsets located along the horizontal and elevation axes at the detector plane, which are expressed as non-zero mean Gaussian distributed random variables, i.e., $R_{x,\{i, j\}} \sim N\left(\mu_{x,\{i, j\}}, \sigma_{x,\{i, j\}}^{2}\right)$ and $R_{y,\{i, j\}} \sim N\left(\mu_{y,\{i, j\}}, \sigma_{y,\{i, j\}}^{2}\right)$, where the parameters $\mu_{x,\{i, j\}}, \mu_{y,\{i, j\}}$, represent their corresponding mean values, and $\sigma_{x,\{i, j\}}, \sigma_{y,\{i, j\}}$, the corresponding jitters for horizontal and elevation displacements, respectively. The joint standard deviation of $\sigma_{x,\{i, j\}}, \sigma_{y,\{i j\}}$, in the $j$ th hop in the $i$ th path, can be obtained as [19],

$$
\sigma_{\bmod ,\{i, j\}}^{2}=\left(\frac{3 \mu_{x,\{i, j\}}^{2} \sigma_{x,\{i, j\}}^{4}+3 \mu_{y,\{i, j\}}^{2} \sigma_{y,\{i, j\}}^{4}+\sigma_{x,\{i, j\}}^{6}+\sigma_{y,\{i, j\}}^{6}}{2}\right)^{1 / 3}
$$

Similarly, the PDF for $I_{p,\{i, j\}}$ can be approximated as [19],

$$
f_{I_{p,\{i, j\}}}\left(I_{p,\{i, j\}}\right)=\frac{\psi_{\{i, j\}}^{2} g_{\{i, j\}\}}^{-\psi_{\{i, j\}}^{2}}}{A_{0,\{i, j\}}^{2}} I_{p,\{i, j\}}^{2} \psi_{\{i, j\}}^{2}-1,0 \leq I_{p,\{i, j\}} \leq g_{\{i, j\}} A_{0,\{i, j\}}
$$

where $\psi_{\{i, j\}}=w_{z, e q,\{i, j\}} / 2 \sigma_{\bmod ,\{i, j\}}, \quad \psi_{x,\{i, j\}}=w_{z, e q,\{i, j\}} / 2 \sigma_{x,\{i, j\}}$ and $g_{\{i, j\}}=$ $\exp \left(\frac{1}{\psi_{\{i, j\}}^{2}}-\frac{1}{\psi_{x,\{i, j\}}^{2}}-\frac{1}{\psi_{y,\{i, j\}}^{2}}-\frac{\mu_{x,\{i, j\}}^{2}}{2 \sigma_{x,\{i, j\}}^{2} \psi_{x,\{i, j\}}^{2}}-\frac{\mu_{y,\{i, j\}}^{2}}{2 \sigma_{y,\{i, j\}}^{2} \psi_{y,\{i, j\}}^{2}}\right)[8,9,17,19,29]$. It should be noted that increased values of the parameter $\psi_{\{i, j\}}$ correspond to a weaker amount of misalignment-induced 
fading, while, $w_{z, e q,\{i, j\}}$ denotes the equivalent beam radius in the $j$ th hop in the $i$ th path, which is given as $w_{z, e q,\{i, j\}}=\left[\sqrt{\pi} \operatorname{erf}\left(v_{\{i, j\}}\right) w_{z,\{i, j\}}^{2} / 2 v_{\{i, j\}} \exp \left(-v_{\{i, j\}}^{2}\right)\right]^{1 / 2}$, where $v_{\{i, j\}}=\sqrt{\pi} r_{a,\{i, j\}} / \sqrt{2} w_{z,\{i, j\}}$, with $r_{a,\{i, j\}}$ being the radius of the circular detection aperture, and $\operatorname{erf}($.$) , the error function. Additionally$ $A_{0,\{i, j\}}$ describes the fraction of the collected power at $r_{a,\{i, j\}}=0$, with $A_{0,\{i, j\}}=\operatorname{erf}^{2}\left(v_{\{i, j\}}\right)[7,19]$. It is also worth mentioning that by setting in the $j$ th hop in the $i$ th path, the boresight displacement is equal to zero, i.e., $s_{\{i, j\}}=\sqrt{\mu_{x,\{i, j\}}^{2}+\mu_{y,\{i, j\}}^{2}}=0$, the Beckmann's distribution reduces to the well-known Rayleigh's distribution in [7], which accurately describes the corresponding zero boresight pointing errors effect [8]. Indeed, for $s_{\{i, j\}}=0$ it holds that $\mu_{x,\{i, j\}}=\mu_{y,\{i, j\}}=0$ and $\sigma_{x,\{i, j\}}=\sigma_{y,\{i, j\}}$ and thus, (6) reduces to Equation (10) in [7] and (8) reduces to Equation (11) in [7].

\subsection{Joint Turbulence and Pointing Errors Effects}

Considering the combined impact of both turbulence-induced and misalignment induced fading, the joint PDF for the normalized irradiance $I_{\{i, j\}}$, is obtained by the following integral $[7,17]$ :

$$
f_{I_{\{i, j\}}}\left(I_{\{i, j\}}\right)=\int f_{I_{\{i, j\}} \mid I_{a,\{i, j\}}}\left(I_{\{i, j\}} \mid I_{a,\{i, j\}}\right) f_{I_{a,\{i, j\}}}\left(I_{a,\{i, j\}}\right) d I_{a,\{i, j\}}
$$

where $f_{I_{\{i, j\}} \mid I_{a,\{i, j\}}}\left(I_{\{i, j\}} \mid I_{a,\{i, j\}}\right)$ is the conditional probability given, $I_{a,\{i, j\}}$, either for G-G or NE distribution, Equations (3) or (5), respectively.

Thus, by using (3) (Equation (07.34.03.060.501) in [30]) and by performing the analysis in [7] we obtain that for weak to strong G-G modeled turbulence, Equation (9) is written as [7,31]:

$$
\begin{aligned}
& f_{G G, I_{\{i, j\}}}\left(I_{\{i, j\}}\right)=\frac{\alpha \beta \psi_{\{i, j\}}^{2}}{A_{0,\{i, j\}} g_{\{i, j\}} \Gamma(\alpha) \Gamma(\beta)}
\end{aligned}
$$

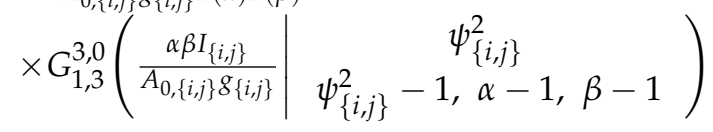

where G[.] denotes the Meijer function, (Equation (5) in [32]) that is a standard built in function which can be evaluated with most of the well known mathematical software packages and can be transformed to the familiar hypergeometric functions [32].

Similarly, for saturated turbulence conditions, by using (5), Equation (11) in [32] and by following the same analysis performed in [7], we obtain that (9) can be written according to [33], as

$$
\begin{aligned}
f_{N E, I_{\{i, j\}}}\left(I_{\{i, j\}}\right) & =\frac{\psi_{\{i, j\}}^{2}}{A_{0,\{i, j\}} g_{\{i, j\}}} \\
& \times G_{1,2}^{2,0}\left(\frac{I_{\{i, j\}}}{A_{0,\{i, j\}} g_{\{i, j\}}} \mid \begin{array}{c}
\psi_{\{i, j\}}^{2} \\
\psi_{\{i, j\}}^{2}-1,0
\end{array}\right)
\end{aligned}
$$

Moreover, the instantaneous electrical SNR at the receiver through $j$ th hop of the $i$ th path is expressed as $\gamma_{\{i, j\}}=2 P^{2} \eta^{2} I_{\{i, j\}}^{2} / \sigma_{n}^{2}$ with the corresponding average electrical SNR being $\mu_{\{i, j\}}=$ $2 P^{2} \eta^{2}\left(E\left[I_{\{i, j\}}\right]\right)^{2} / \sigma_{n}^{2}$, where $P$ denotes the average signal power at the receiver's side and $E\left[I_{\{i, j\}}\right]$ is the corresponding expected normalized irradiance value, $[15,17,22]$ which for both G-G and NE turbulence distributions is given according to [33-35], as:

$$
E\left[I_{\{i, j\}}\right]=A_{\psi,\{i, j\}}=A_{0,\{i, j\}} g_{\{i, j\}}\left(1+\psi_{\{i, j\}}^{-2}\right)^{-1}
$$

\section{Outage Probability Estimation}

In order to evaluate the performance of the examined system, we estimate its $\mathrm{OP}$, which is a crucial performance metric, in terms of reliability, for any communication system. This metric represents the 
probability that the instantaneous electrical SNR at the receiver falls below a critical threshold value, $\gamma_{t h}$, which corresponds to the receiver's sensitivity limit. Therefore, the OP inthe $j$ th hop in the $i$ th path is expressed as $[10,12,15,17]$ :

$$
P_{\text {out },\{i, j\}}=\operatorname{Pr}\left(I_{\{i, j\}}<I_{\{i, j\}, t h}\right)=F_{I_{\{i, j\}}}\left(I_{\{i, j\}, t h}\right)=\int_{0}^{I_{\{i, j\}, t h}} f_{I_{\{i, j\}}}\left(I_{\{i, j\}}\right) d I_{\{i, j\}}
$$

where $\operatorname{Pr}($.$) denotes probability, f_{I_{\{i, j\}}}\left(I_{\{i, j\}}\right)$ stands for the probability density function (PDF) either of the G-G or the NE turbulence distribution, and $F_{I_{\{i, j\}}}\left(I_{\{i, j\}, t h}\right)$ represents the corresponding cumulative density function (CDF) of the G-G or the NE turbulence distribution, respectively.

Assuming G-G modeled turbulence, from (10), (12) and (13) (Equation (07.34.17.0011.01) in [30], Equation (26) in [32]) and by setting then $I_{\{i, j\}}=A_{\psi, t h} \sqrt{\gamma_{\{i, j\}, t h} / \mu_{\{i, j\}}}$, [4], where $\gamma_{\{i, j\}, t h} / \mu_{\{i, j\}}$ represents the normalized average electrical SNR, we conclude that (13) can be written as:

$$
\begin{aligned}
P_{G G, o u t,\{i, j\}} & =\frac{\psi_{\{i, j\}}^{2}}{\Gamma(\alpha) \Gamma(\beta)} \\
& \times G_{2,4}^{3,1}\left(\frac{\alpha \beta \psi_{\{i, j\}}^{2}}{\psi_{\{i, j\}}^{2}+1} \sqrt{\frac{\gamma_{t h}}{\mu}} \mid \begin{array}{c}
1, \psi_{\{i, j\}}^{2} \\
\psi_{\{i, j\}}^{2}, \alpha, \beta, 0
\end{array}\right)
\end{aligned}
$$

Alternatively, by performing once again the procedure above but by using (11) instead of (10), we conclude that for NE modeled turbulence, (13) gives the following expression:

$$
P_{N E, \text { out },\{i, j\}}=\psi_{\{i, j\}}^{2} G_{2,3}^{2,1}\left(\frac{\psi_{\{i, j\}}^{2}}{\psi_{\{i, j\}}^{2}+1} \sqrt{\frac{\gamma_{t h}}{\mu}} \mid \begin{array}{c}
1, \psi_{\{i, j\}}^{2}+1 \\
\psi_{\{i, j\}}^{2} 1,0
\end{array}\right)
$$

Note that contrary to [4] that evaluates the average bit error rate (ABER) as a function of the average SNR, $\mu$, in this work we evaluate through (14) and (15) the OP as a function of the normalized average electrical SNR $\gamma_{\{i, j\}, t h} / \mu_{\{i, j\}}$ that is assumed to obtain the same value for each link of the examined FSO system, i.e., $\gamma_{\{i, j\}, t h} / \mu_{\{i, j\}}=\gamma_{t h} / \mu$, under G-G and NE modeled turbulence conditions, respectively. Moreover, (14) and (15) refer to mixed DF relay FSO configurations, and thus, contrary to the ABER expressions in [4] they introduce the indices $i$ and $j$.

The OP of each multi-hop branch of the system, i.e., the OP of each parallel link of serially connected DF relays of the $i$ th path is given as $[10,17]$ :

$$
P_{\text {out }, i}=1-\prod_{j=1}^{H}\left[1-P_{\text {out },\{i, j\}}\right]
$$

and taking into account that $N$ parallel links are used the total OP of the system is written as $[10,17]$ :

$$
P_{\text {out }}=\prod_{i=1}^{N} P_{\text {out }, i}=\prod_{i=1}^{N}\left\{1-\prod_{j=1}^{H}\left[1-P_{\text {out },\{i, j\}}\right]\right\}
$$

from (12) and (14), the following closed-form mathematical expression for the OP of the total system under G-G modeled turbulence along with non-zero boresight pointing errors, is obtained:

$$
P_{G G, \text { out }}=\prod_{i=1}^{N}\left\{1-\prod_{j=1}^{H}\left[1-\frac{\psi_{\{i, j\}}^{2}}{\Gamma(\alpha) \Gamma(\beta)} G_{2,4}^{3,1}\left(\sqrt{\frac{\gamma_{\{i, j\}, t h}}{\mu_{\{i, j\}}}} \frac{\alpha \beta \psi_{\{i, j\}}^{2}}{\psi_{\{i, j\}}^{2}+1} \mid \begin{array}{c}
1, \psi_{\{i, j\}}^{2} \\
\psi_{\{i, j\}}^{2}, \alpha, \beta, 0
\end{array}\right)\right]\right\}
$$


Similarly, from (12), (16), (17) and by using (15), the following closed-form mathematical expression for the OP of the total system under NE modeled turbulence along with non-zero boresight pointing errors, is derived:

$$
P_{N E, \text { out }}=\prod_{i=1}^{N}\left\{1-\prod_{j=1}^{H}\left[1-\psi_{\{i, j\}}^{2} G_{2,3}^{2,1}\left(\sqrt{\frac{\gamma_{\{i, j\}, t h}}{\mu_{\{i, j\}}}} \frac{\psi_{\{i, j\}}^{2}}{\psi_{\{i, j\}}^{2}+1} \mid \begin{array}{c}
1, \psi_{\{i, j\}}^{2}+1 \\
\psi_{\{i, j\}}^{2} 1,0
\end{array}\right)\right]\right\}
$$

\section{Mean Outage Duration Estimation}

In the context of outage performance estimation, another metric, relevant to outage probability at least as important, and very practical, is the mean outage duration (MOD) of the FSO system. Indeed, the value of MOD is a significant figure of merit, because in combination with the throughput of the communication system, it relates to the percentage of information loss over a properly selected reference time. In fact, this metric denotes the average duration over the reference time interval that the examined communication system fails to operate properly. Thus, MOD indicates the average period of time that an outage occurs, i.e., the average duration that the system is practically unavailable over the determined reference time. Consequently, it becomes evident, that MOD is of particular practical use for FSO systems design, since its value directly reveals whether the investigated system can meet the required outage specifications or not. More precisely, the MOD, $T_{o d}$ for a specific period of time is calculated as $[29,36]$ :

$$
T_{\text {od }}=P_{\text {out }} T_{R}
$$

with $T_{R}$ being the appropriately chosen reference time, e.g., $T_{R}=3600 \mathrm{~s}$ for the case where the reference time is one hour. Additionally, the coherence time of the atmospheric turbulence, $\tau_{0}$, is in the range of milliseconds [37,38], and for $T_{o d}$, it should be verified that $T_{o d} \geq \tau_{0}$. Furthermore, note that for the simulation experiments conducted in the next section we assume a reference time of one hour.

In view of the above, by substituting either (18) or (19) into (20) we obtain the closed-form expression for the MOD valueof the total FSO system, $T_{G G, o d}$, for G-G:

$$
T_{G G, o d}=T_{R}\left\{\prod_{i=1}^{N}\left\{1-\prod_{j=1}^{H}\left[1-\frac{\psi_{\{i, j\}}^{2}}{\Gamma(\alpha) \Gamma(\beta)} G_{2,4}^{3,1}\left(\sqrt{\frac{\gamma_{\{i, j\}, t h}}{\mu_{\{i, j\}}}} \frac{\alpha \beta \psi_{\{i, j\}}^{2}}{\psi_{\{i, j\}}^{2}+1} \mid \begin{array}{c}
1, \psi_{\{i, j\}}^{2} \\
\psi_{\{i, j\}}^{2}, \alpha, \beta, 0
\end{array}\right)\right]\right\}\right\}
$$

or, $T_{N E, o d}$, for NE turbulent channels, respectively, as:

$$
T_{N E, o d}=T_{R}\left\{\prod_{i=1}^{N}\left\{1-\prod_{j=1}^{H}\left[1-\psi_{\{i, j\}}^{2} G_{2,3}^{2,1}\left(\sqrt{\frac{\gamma_{\{i, j\}, t h}}{\mu_{\{i, j\}}}} \frac{\psi_{\{i, j\}}^{2}}{\psi_{\{i, j\}}^{2}+1} \mid \begin{array}{c}
1, \psi_{\{i, j\}}^{2}+1 \\
\psi_{\{i, j\}}^{2}, 1,0
\end{array}\right)\right]\right\}\right\}
$$

\section{Numerical Results}

In this section we present the numerical results concerning both the OP and MOD performance of the examined relay-assisted FSO system, for weak to saturated turbulence along with different amounts of pointing mismatch, over a wide SNR range. Specifically, by using (18) and (19) we obtain the numerical results concerning the $\mathrm{OP}$ of the system for gamma-gamma modeled and negative exponential modeled turbulence conditions, respectively, along with the presence of non-zero boresight pointing errors. Similarly, by using (21) and (22) we get the numerical results for the corresponding MOD values of the system, i.e., for G-G and NE turbulence, respectively, along with non-zero boresight misalignment-induced fading.

The relay-aided FSO system under investigation is assumed to operate at $\lambda=1.55 \mu \mathrm{m}$ and receiver's aperture diameter, $D=0.1 \mathrm{~m}$. Additionally, each hop length of the system, $L$, is $1.5 \mathrm{~km}$, while the system employs $N=1,2$ or 5 parallel DF relays and $H=2$ or 4 hops. For G-G modeled, weak turbulence conditions, the parameter $C_{n}{ }^{2}$ value is fixed at $2 \times 10^{-14} \mathrm{~m}^{-2 / 3}$, while for NE modeled, saturated turbulent channels, the parameter $C_{n}{ }^{2}$ obtains its maximum value, i.e., $C_{n}{ }^{2}=10^{-13} \mathrm{~m}^{-2 / 3}$. 
Concerning the point errors effect, the boresight is zero only for $N=1$ due to precise positioning, [4]. In that case it holds that $\left(\mu_{x} / r_{a}, \mu_{y} / r_{a}\right)=(0,0)$ and $\sigma_{x}=\sigma_{y}$, while $\psi_{1}=5$ for $\left(r_{a}, w_{z} / r_{a}, \mu_{x} / r_{a}, \mu_{y} / r_{a}, \sigma_{x} / r_{a}, \sigma_{y} / r_{a}\right)=(5 \mathrm{~cm}, 10,0,0,1,1)$. When $N=3$ relays are considered, we assume for the remaining paths that $\left(r_{a}, w_{z} / r_{a}, \mu_{x} / r_{a}, \mu_{y} / r_{a}\right)=(5 \mathrm{~cm}, 10,1,2)$, while for weak to strong non-zero boresight pointing errors, the parameter $\psi_{2}$ takes the value 2.3 or 1.3 for $\left(\sigma_{x} / r_{a}, \sigma_{y} / r_{a}\right)$ $=(2.1,1.5)$ or $\left(\sigma_{x} / r_{a}, \sigma_{y} / r_{a}\right)=(4,3)$, respectively. Finally, for $N=5$, the non-zero boresight pointing errors effect is stronger and even more stronger, with $\psi_{3}=0.9$ or $\psi_{3}=0.6$, for $\left(\sigma_{x} / r_{a}, \sigma_{y} / r_{a}\right)=(5.8,4.8)$ or $\left(\sigma_{x} / r_{a}, \sigma_{y} / r_{a}\right)=(9,7)$, respectively.

Figure 2, illustrates the evolution of OP performance over a wide normalized average electrical SNR range, obtained by using (19), for the total relay-assisted examined FSO system when it employs $N=1,3$ or 5 dual-hop paths, i.e., $H_{j}=2$, under saturated turbulence conditions, modeled through the NE distribution, along with zero boresight pointing errors for the single path configuration, i.e., for $N=1$, and additional weak to strong non-zero boresight pointing errors for multiple path configurations, i.e., for $N=3$ or 5 . It is clearly shown that even in saturated turbulence conditions, the OP performance is significantly improved by using parallel relaying method with dual-hop paths, and especially, by increasing the number of paths of the total system. Additionally, further OP performance enhancements are depicted as the normalized average electrical SNR obtains larger values and as the non-zero boresight misalignment-induced fading gets weaker, i.e., for larger $\psi_{2}$ or $\psi_{3}$ parameter values that refer to triple-path $(N=3)$ and quintuple-path $(N=5)$ dual-hop implementations, respectively. Moreover, it is worth mentioning that the performance comparison between the corresponding curves of Figure 2 and those which appear in Ref. [17] obtained by the analytical expression Equation (15) in [17], demonstrates significantly larger OP corresponding values due to the fact that Figure 2 refers to saturated NE modeled turbulence channels, while the results of [17] have been obtained for weak turbulence conditions with a different model, i.e. the Gamma distribution.

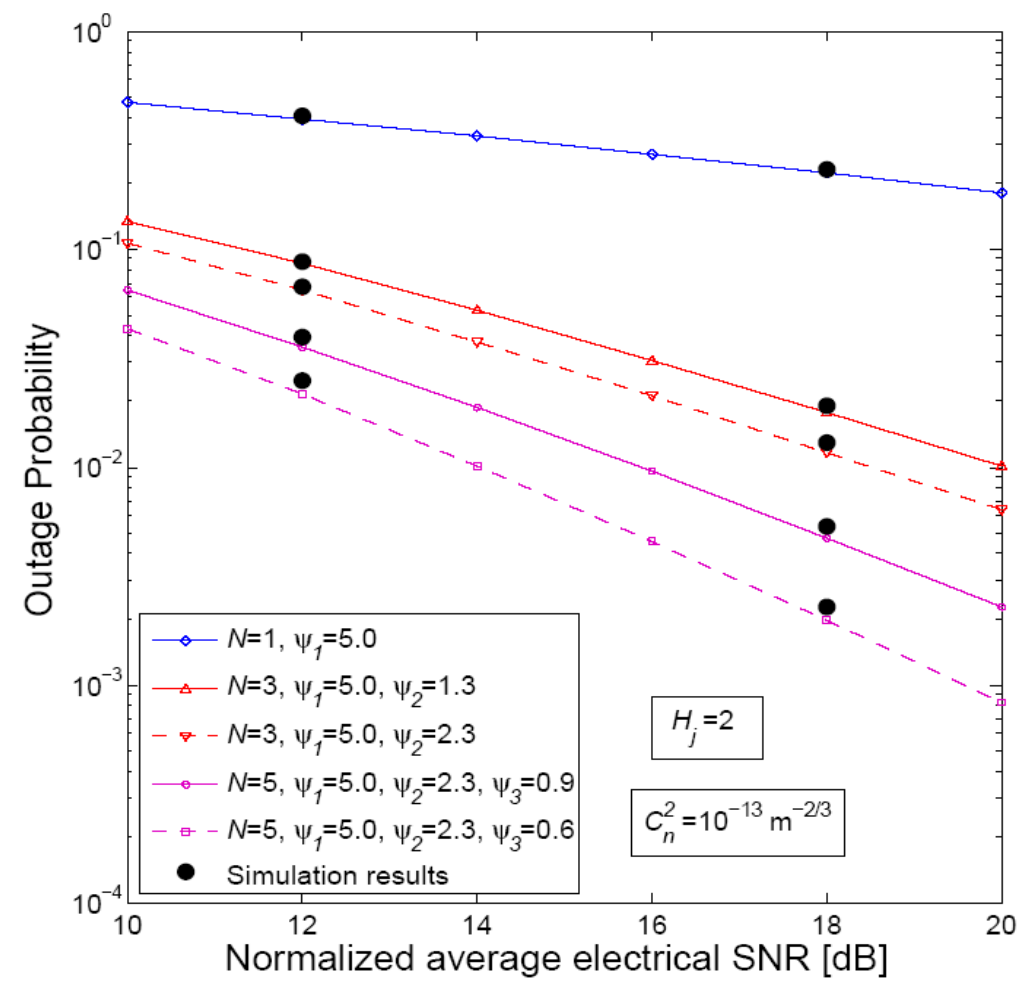

Figure 2. Total outage probability (OP) of a dual-hop relay-aided system with varrying path configurations as a function of a wide range of $\gamma / \mu$, for negative exponential (NE) modeled saturated turbulent channels along with different amounts of pointing mismatch. 
In Figure 3, the corresponding results with those of Figure 2 are presented, but for $H_{j}=4$. It can be seen that although qualitatively behavior is the same for both cases, in Figure 3 degraded corresponding OP performance results are obtained due to the larger number of hops in each path that lead to longer path lengths. However, we ought to mention that by increasing the hops number, the coverage area also increases. Thus, at the expense of an increase in OP values we can broaden the coverage FSO area, while considering our system's OP specifications and demands, we can conclude whether it is wise to extend the total coverage area of our FSO system, under specific turbulence-induced and misalignment-induced fading conditions. Furthermore, by comparing Figures 2 and 3 in [17] obtained by the analytical expression Equation (15) in [17], Figure 3 depicts significantly increased OP corresponding values due to the stronger turbulence conditions that are assumed.

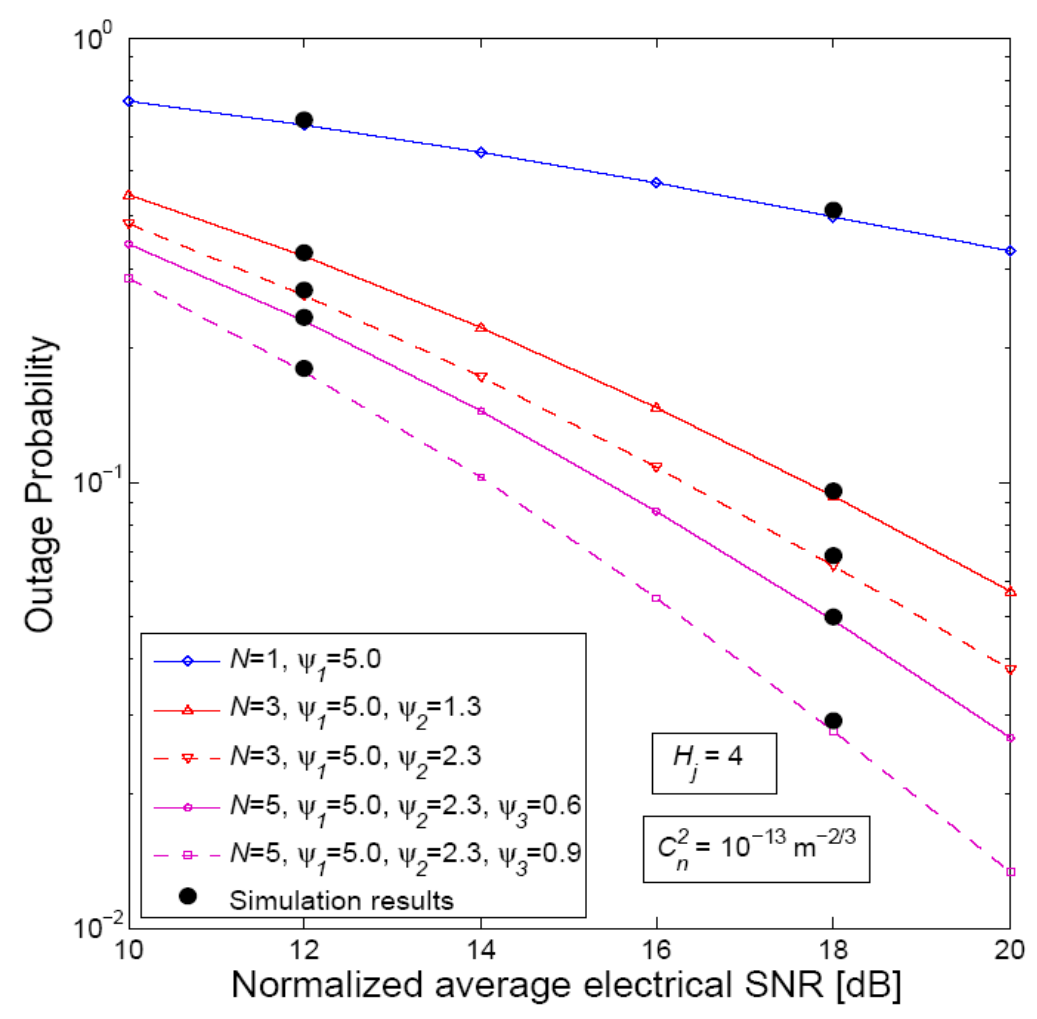

Figure 3. Total outage probability (OP) of a quadruple-hop relay-aided system with varying path configurations as a function of a wide range of $\gamma / \mu$, for negative exponential (NE) modeled saturated turbulent channels along with different amounts of pointing mismatch.

Figure 4 shows the evolution of OP performance over a wide normalized average electrical SNR range, obtained by using (19), for the total relay-assisted examined FSO system when it employs $N=1,3$ or 5 dual-hop paths, i.e., $H_{j}=2$, under weak turbulence conditions, modeled through the G-G distribution, along with zero boresight pointing errors for $N=1$, and additional weak to strong non-zero boresight pointing errors for $N=3$ or 5 . Thus, the performance comparison between Figures 2 and 4 demonstrates that the obtained results of the latter outperformed the corresponding results of the former, in terms of OP. This is due to a weaker amount of turbulence strength that the FSO propagation has to deal with in the latter case. Consequently, the comparison between these two figures reveals the detrimental impact of turbulence-induced fading, on the outage FSO performance. 


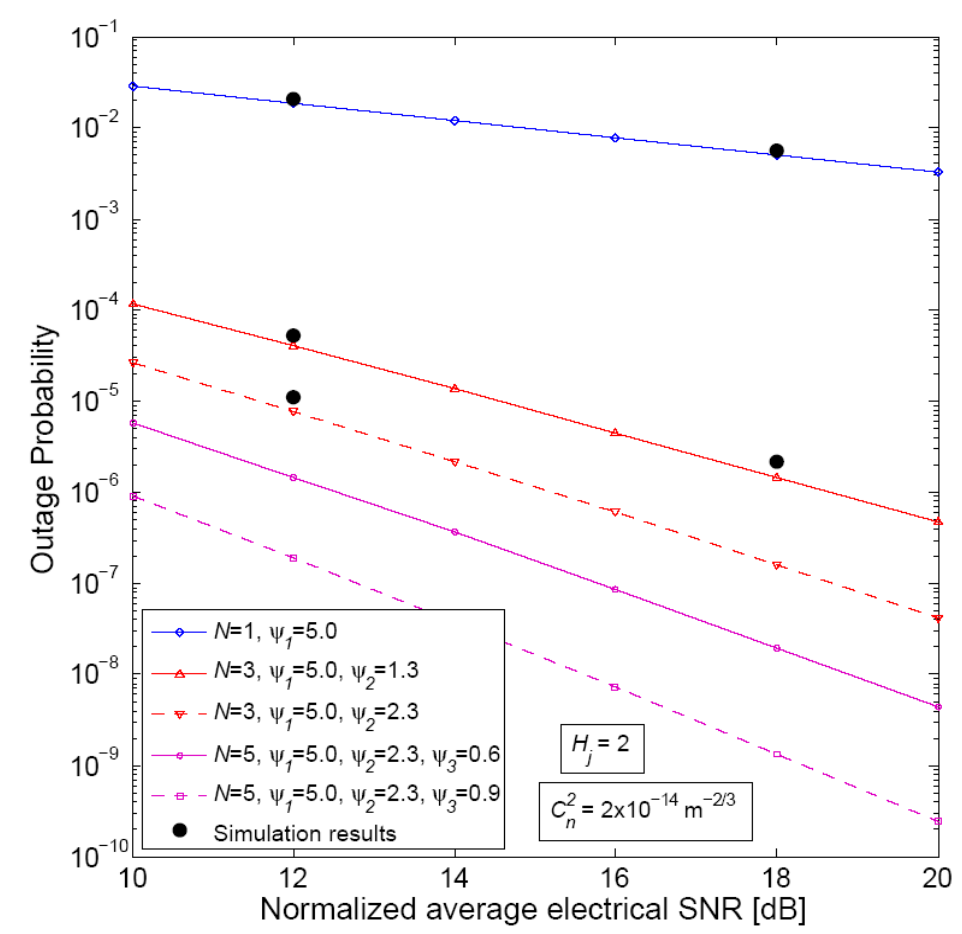

Figure 4. Total outage probability (OP) of a dual-hop relay-aided system with varying path configurations as a function of a wide range of $\gamma / \mu$, for gamma-gamma (G-G) modeled weak turbulent channels, along with different amounts of pointing mismatch.

In Figure 5, the corresponding results of those of Figure 4 are shown, but for $H_{j}=4$. Once again, despite the identical qualitative behavior of the two figures, we now obtain increased corresponding OP values due to the larger number of the serially connected DF relays that are employed in each path. Furthermore, due to the weak turbulence conditions instead of the saturated we investigated before, we can observe the current performance comparison, i.e., between Figures 4 and 5, which shows less significant outage performance degradation, by doubling the hops in each path, than the corresponding performance comparison between the Figures 2 and 3.

Figure 6, depicts the evolution of MOD values over a wide normalized average electrical SNR range, obtained by using (22), for the total relay-aided FSO system with $N=1,3$ or 5 quadruple-hop paths, under negative exponential modeled saturated turbulence along with varying non-zero boresight pointing errors effects for multi-path configurations. It becomes evident that for a specific normalized average electrical SNR value the corresponding MOD value is significantly reduced by increasing the number of paths, even in saturated turbulence, along with strong pointing errors. Indeed, we can observe that for a normalized average electrical SNR, $\gamma / \mu=18 \mathrm{~dB}$, the MOD value per hour is shown to be almost equal to $23.8 \mathrm{~min}$ for the single-path configuration, while for $N=5$ the MOD value is reduced almost to $2.9 \mathrm{~s}$ or $1.6 \mathrm{~s}$ for strong and weak non-zero boresight pointing errors, respectively. Moreover, as it was expected, the MOD value of the system increases for lower normalized average electrical SNR values and stronger non-zero boresight amounts of pointing mismatch. 


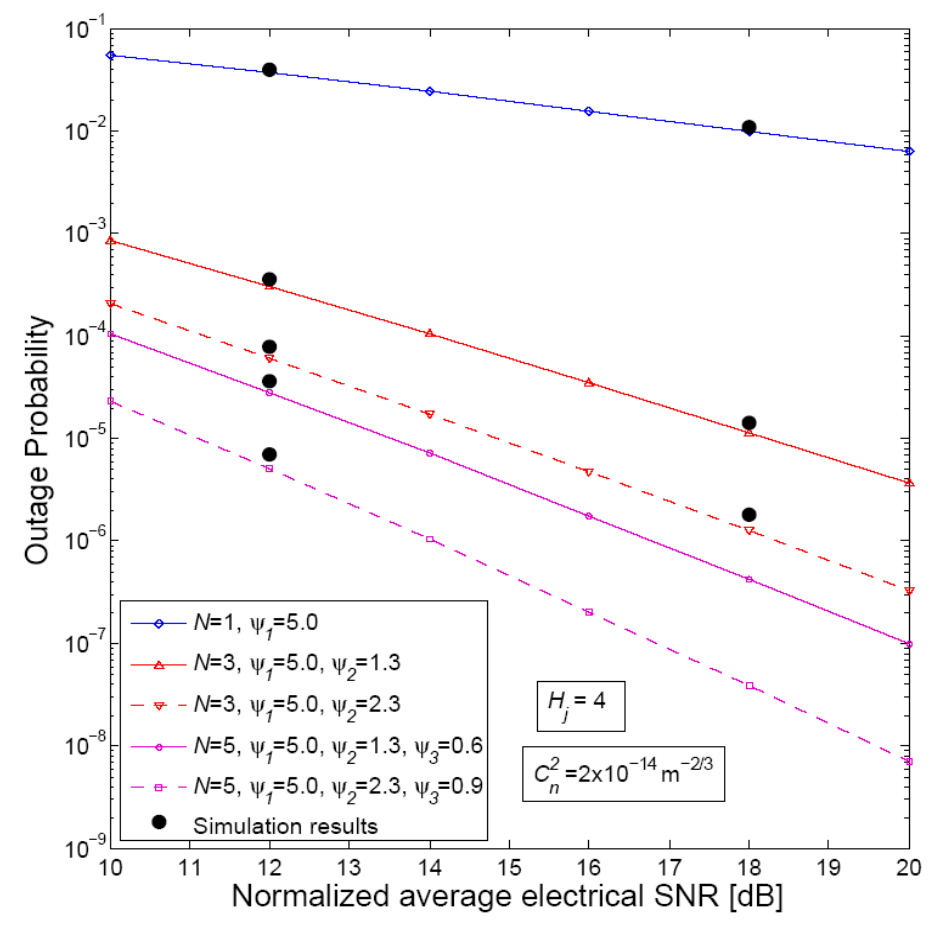

Figure 5. Total outage probability (OP) of a quadruple-hop relay-aided system with varying path configurations as a function of a wide range of $\gamma / \mu$, for gamma-gamma (G-G) modeled weak turbulent channels along with different amounts of pointing mismatch.

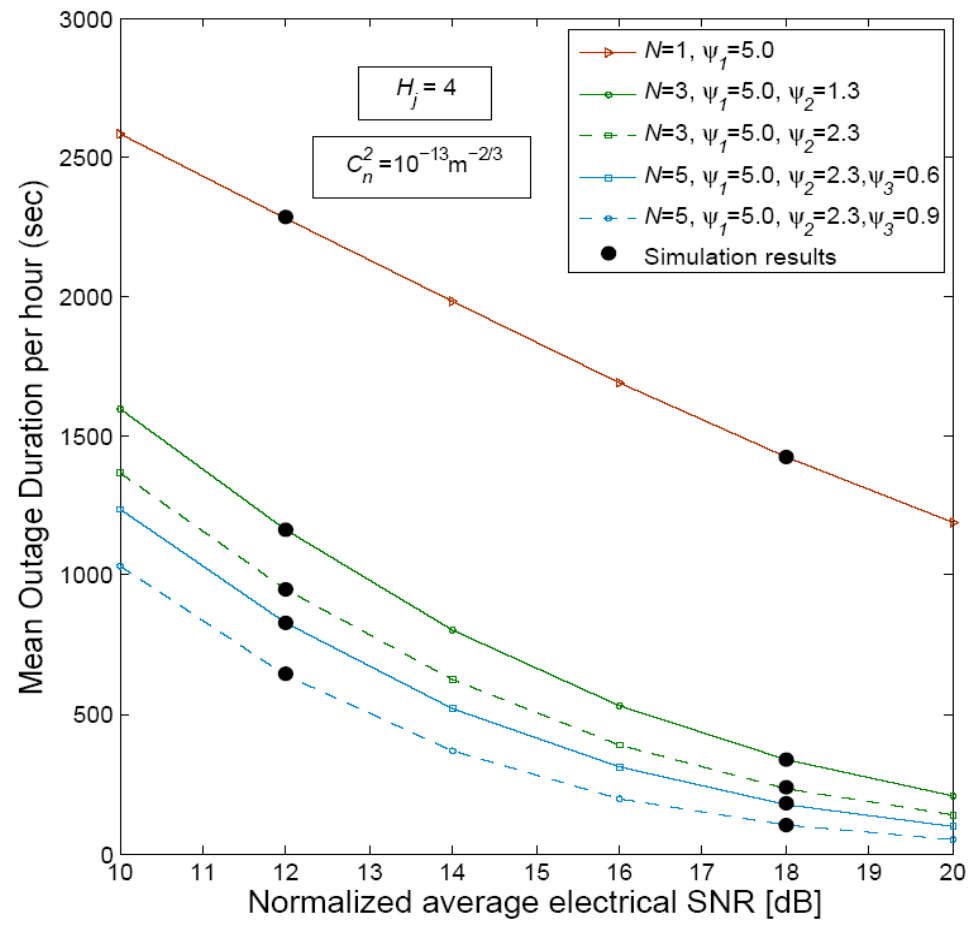

Figure 6. Total mean outage duration (MOD) of a quadruple-hop relay-aided system with varying path configurations as a function of a wide range of $\gamma / \mu$, for negative exponential (NE) modeled saturated turbulent channels along with different amounts of pointing mismatch.

Figure 7, highlights the evolution of MOD values per hour over a wide normalized average electrical SNR range, obtained by using (21), for the total relay-aided FSO system with $N=3$ or 5 and $H_{j}=2$ or 4 , under weak gamma-gamma modeled turbulence and different amounts of non-zero 
boresight pointing errors. As we can see, apart from increasing the electrical SNR value and mitigating the strength of pointing errors effects, the increase of the number of paths can lead to significant outage performance enhancements of the total system, and therefore, $N=5$ configurations are shown to outperform $N=3$ configuration, in terms of MOD metric. However, by increasing also the number of hops in each path, the total MOD value increases too. Additionally, the performance comparison between Figures 6 and 7 concludes that the MOD results of the latter outperform the corresponding MOD results of the former. This is due to the weaker amount of turbulence-induced fading that is addressed in the latter case. In fact, given that $\gamma / \mu=18 \mathrm{~dB}$ for $N=5$ with $H_{j}=4$ and strong non-zero boresight pointing errors, due to the weaker turbulence conditions we now obtain a strongly reduced MOD value almost of $1.5 \mathrm{~ms}$ per hour instead of the almost $2.9 \mathrm{~s}$ per hour we obtained before for the same system's characteristics, except for saturated turbulence conditions. Additionally, given that $\gamma / \mu=18 \mathrm{~dB}$ for $N=5$ with $H_{j}=4$ and weak non-zero boresight pointing errors this time, the MOD value is now shown to be almost $4.8 \mu$ s per hour instead of the corresponding MOD value of almost $1.6 \mathrm{~s}$ per hour of Figure 6.

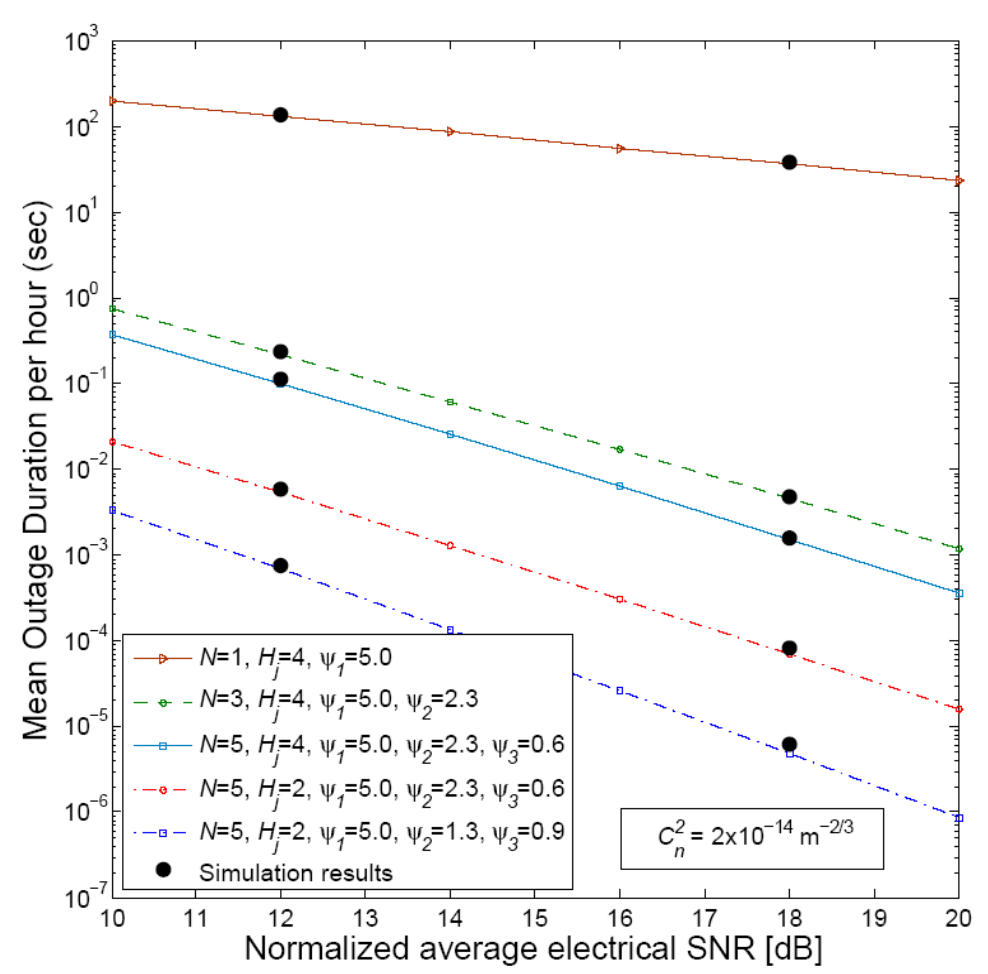

Figure 7. Total mean outage duration time (MOD) of a relay-aided system with both varying path and hop configurations as a function of a wide range of $\gamma / \mu$, for gamma-gamma (G-G) modeled weak turbulent channels along with different amounts of pointing mismatch.

\section{Conclusions}

In this work, a flexible mixed parallel and muliti-hop DF relaying FSO architecture has been introduced in order to effectively combat weak to saturated atmospheric turbulence effects, along with weak to strong non-zero boresight pointing errors, which significantly mitigate the FSO outage performance and availability. In this context, novel closed form mathematical expressions have been derived for both OP and MOD metrics over NE and G-G modeled turbulence, along with Beckman modeled non-zero boresight pointing errors modeled. By using these expressions, proper numerical results are presented which are further validated by the corresponding simulations. Specifically, it has been demonstrated that by increasing the number of paths of the system, i.e., the number of parallel connected DF relays, significant outage performance improvements can be achieved. Moreover, it has been shown that the outage performance of such a system can be further upgraded but at the expense 
of its total coverage area, i.e., by decreasing the number of hops in each path, namely the number of the serially connected DF relays. Consequently, by considering the required technical specifications for the FSO system under design and by using the extracted expressions, we may find the optimal selection for the exact number of parallel and serially connected DF relays, and thus, we may optimally balance any contradicting requirements of coverage area broadening and outage performance enhancement. Thus, the analysis proposed in this work, is a very useful tool for the design, the flexibility, the scalability, the outage performance and the availability of FSO systems.

Author Contributions: Conceptualization, G.K.V., H.E.N. and A.N.S.; methodology, G.K.V. and H.E.N.; validation, G.K.V., A.N.S., C.K.V. and V.C.; formal analysis, G.K.V., H.E.N., G.S.T. and C.K.V.; investigation, G.K.V., H.E.N., A.N.S. and V.C.; writing-original draft preparation, G.K.V.; writing-review and editing, H.E.N. and G.S.T.; supervision, H.E.N., G.S.T.

Funding: This research has received funding from the European Union's Horizon 2020 research and innovation program under grant agreement No: 777596.

Conflicts of Interest: The authors declare no conflict of interest.

\section{References}

1. Ghassemlooy, Z.; Popoola, W.O. Terrestrial Free-Space Optical Communications. In Mobile and Wireless Communications: Network Layer and Circuit Level Design; Fares, S.A., Adachi, F., Eds.; InTech: London, UK, 2010; pp. 355-392.

2. Henniger, H.; Wilfert, O. An Introduction to Free-Space Optical Communications. Radioengineering 2010, 19, 203-212.

3. Varotsos, G.K.; Nistazakis, H.E.; Volos, C.K.; Tombras, G.S. FSO links with diversity pointing errors and temporal broadening of the pulses over weak to strong atmospheric turbulence channels. Elsevier Opt. 2016, 127, 3402-3409. [CrossRef]

4. Varotsos, G.K.; Nistazakis, H.E.; Petkovic, M.I.; Djordjevic, G.T.; Tombras, G.S. SIMO optical wireless links with nonzero boresight pointing error over M modeled turbulence channels. Opt. Commun. 2017, 303, 391-400. [CrossRef]

5. Zhu, X.; Kahn, J.M. Free-space optical communication through atmospheric turbulence channels. IEEE Trans. Commun. 2002, 50, 1293-1300.

6. Arnon, S. Effects of atmospheric turbulence and building sway on optical wireles-communication systems. Opt. Lett. 2003, 28, 129-131. [CrossRef] [PubMed]

7. Farid, A.; Hranilovic, S. Outage capacity optimization for free-space optical links with pointing errors. J. Lightwave Technol. 2007, 25, 1702-1710. [CrossRef]

8. Yang, F.; Cheng, J.; Tsiftsis, T.A. Free-space optical communication with nonzero boresight pointing errors. IEEE Trans. Commun. 2014, 62, 713-725. [CrossRef]

9. Boluda-Ruiz, R.; Garcia-Zambrana, A.; Castillo-Vazquez, B.; Castillo-Vazquez, C. Impact of nonzeroboresight pointing error on ergodic capacity of MIMO FSO communication systems. Opt. Express 2016, 24, 3513-3534. [CrossRef] [PubMed]

10. Prabu, K.; Kumar, D.S. Outage Analysis of Relay-Assisted BPSK-SIM Based FSO Systems over Strong Atmospheric turbulence with Pointing errors. Int. J. Comput. Commun. Eng. 2014, 3, 317-320. [CrossRef]

11. Acampora, A.S.; Krishnamurthy, S.V. A broadband wireless access network based on mesh-connected free-space optical links. IEEE Pers. Commun. 1999, 6, 62-65. [CrossRef]

12. Tsiftsis, T.A.; Sandalidis, H.G.; Karagiannidis, G.K.; Sagias, N.C. Multihop free-space optical communications over strong turbulence channels. In Proceedings of the 2006 IEEE International Conference on Communications, Istanbul, Turkey, 11-15 June 2006; Volume 6, pp. 2755-2759.

13. Karagiannidis, G.K.; Tsiftsis, T.A.; Sandalidis, H.G. Outage probability of relayed free space optical communication systems. Electron. Lett. 2006, 42, 994-996. [CrossRef]

14. Safari, M.; Uysal, M. Relay-assisted free-space optical communication. IEEE Trans. Wirel. Commun. 2008, 7, 5441-5449. [CrossRef] 
15. Feng, M.; Wang, J.B.; Sheng, M.; Cao, L.L.; Xie, X.X.; Chen, M. Outage performance for parallel relay-assisted free-space optical communications in strong turbulence with pointing errors. In Proceedings of the 2011 International Conference on Wireless Communications and Signal Processing, Nanjing, China, 9-11 November 2011; pp. 1-5.

16. Wang, P.; Wang, R.; Guo, L.; Cao, T.; Yang, Y. On the performances of relay-aided FSO system over M distribution with pointing errors in presence of various weather conditions. Opt. Commun. 2016, 367, 59-67. [CrossRef]

17. Varotsos, G.K.; Nistazakis, H.E.; Stassinakis, A.N.; Tombras, G.S.; Christofilakis, V.; Volos, C.K. Outage performance of mixed, parallel and serial DF relayed FSO links over weak turbulence channels with nonzero boresight pointing errors. In Proceedings of the 2018 7th International Conference in Modern Circuits and Systems Technologies (MOCAST2018), Thessaloniki, Greece, 7-9 May 2018; p. 4.

18. Epple, B. Simplified channel model for simulation of free-space optical communications. IEEE/OSA J. Opt. Commun. Netw. 2010, 2, 293-304. [CrossRef]

19. Boluda-Ruiz, R.; Garcia-Zambrana, A.; Castillo-Vazquez, C.; Castillo-Vazquez, B. Novel approximation of misalignment fading modeled by Beckmann distribution on free-space optical links. Opt. Express 2016, 24, 22635-22649. [CrossRef]

20. Al-Habash, M.A.; Andrews, L.C.; Phillips, R.L. Mathematical model for the irradiance probability density function of a laser beam propagating through turbulent media. Opt. Eng. 2001, 40, 1554-1562. [CrossRef]

21. Popoola, W.O.; Ghassemlooy, Z.; Ahmadi, V. Performance of sub-carrier modulated free-space optical communication link in negative exponential atmospheric turbulence environment. Int. J. Auton. Adapt. Commun. Syst. 2008, 1, 342-355. [CrossRef]

22. Nistazakis, H.E.; Stassinakis, A.N.; Muhammad, S.S.; Tombras, G.S. BER Estimation for Multi Hop RoFSO QAM or PSK OFDM Communication Systems Over Gamma Gamma or Exponentially Modeled Turbulence Channels. Elsevier Opt. Laser Technol. 2014, 64, 106-112. [CrossRef]

23. Muhammad, S.S.; Köhldorfer, P.; Leitgeb, E. Channel Modeling for Terrestrial Free Optical Links. In Proceedings of the 7th International Conference on Transparent Optical Networks ICTON 2005, Barcelona, Spain, 3-7 July 2005; pp. 407-410.

24. Gappmair, W. Novel results on pulse-position modulation performance for terrestrial free-space optical links impaired by turbulent atmosphere and pointing errors. IET Commun. 2012, 6, 1300-1305. [CrossRef]

25. Gradshteyn, I.S.; Ryzhik, I.M. Table of Integrals, Series, and Products, 6th ed.; Academic: New York, NY, USA, 2008.

26. Gappmair, W.; Nistazakis, H.E. Subcarrier PSK performance in terrestrial FSO links impaired by Gamma-Gamma fading, pointing errors, and phase noise. J. Lightwave Technol. 2017, 35, 1624-1632. [CrossRef]

27. Varotsos, G.K.; Stassinakis, A.N.; Nistazakis, H.E.; Tsigopoulos, A.D.; Peppas, K.P.; Aidinis, C.J.; Tombras, G.S. Probability of fade estimation for FSO links with time dispersion and turbulence modeled with the gamma-gamma or the IK distribution. Opt. Int. J. Light Electron Opt. 2014, 125, 7191-7197. [CrossRef]

28. Garcia-Zambrana, A.; Castillo-Vazquez, B.; Castillo-Vazquez, C. Average Capacity of FSO Links with Transmit Laser Selection Using Non-Uniform OOK Signaling Over Exponential Atmospheric Turbulence Channels. Opt. Express 2010, 18, 20445-20454. [CrossRef] [PubMed]

29. Varotsos, G.K.; Nistazakis, H.E.; Gappmair, W.; Sandalidis, H.G.; Tombras, G.S. DF Relayed Subcarrier FSO Links over Malaga Turbulence Channels with Phase Noise and Non-Zero Boresight Pointing Errors. Appl. Sci. 2018, 8, 664. [CrossRef]

30. The Wolfram Functions Site. 2008. Available online: http:/ functions.wolfram.com (accessed on 20 October 2018).

31. Sandalidis, H.G.; Tsiftsis, T.A.; Karagiannidis, G.K. Optical wireless communications with heterodyne detection over turbulence channels with pointing errors. J. Lightwave Technol. 2009, 27, 4440-4445. [CrossRef]

32. Adamchik, V.S.; Marichev, O.I. The Algorithm for Calculating Integrals of Hypergeometric Type Function and its Realization in Reduce System. In Proceedings of the International Conference on Symbolic and Algebraic Computation, Tokyo, Japan, 20-24 August 1990; pp. 212-224.

33. Ninos, M.P.; Nistazakis, H.E.; Tombras, G.S. On the BER performance of FSO links with multiple receivers and spatial jitter over gamma-gamma or exponential turbulence channels. Opt. Int. J. Light Electron Opt. 2017, 138, 269-279. [CrossRef] 
34. Ansari, I.S.; Yilmaz, F.; Alouini, M.S. Performance Analysis of Free-Space Optical Links Over Málaga Turbulence Channels with Pointing Errors. IEEE Trans. Wirel. Commun. 2016, 15, 91-102. [CrossRef]

35. Djordjevic, G.T.; Petkovic, M.I. Average BER performance of FSO SIM-QAM systems in the presence of atmospheric turbulence and pointing errors. J. Mod. Opt. 2016, 63, 715-723. [CrossRef]

36. Proakis, J.G. Digital Communications; McGraw-Hill: New York, NY, USA, 1989.

37. Davis, J.; Tango, W. Measurement of the atmospheric coherence time. Publ. Astron. Soc. Pac. 1996, $108,456$. [CrossRef]

38. Popoola, W.O.; Ghassemlooy, Z.; Lee, C.G.; Boucouvalas, A.C. Scintillation effect on intensity modulated laser communication systems-A laboratory demonstration. Opt. Laser Technol. 2010, 42, 682-692. [CrossRef]

2018 by the authors. Licensee MDPI, Basel, Switzerland. This article is an open access article distributed under the terms and conditions of the Creative Commons Attribution (CC BY) license (http://creativecommons.org/licenses/by/4.0/). 